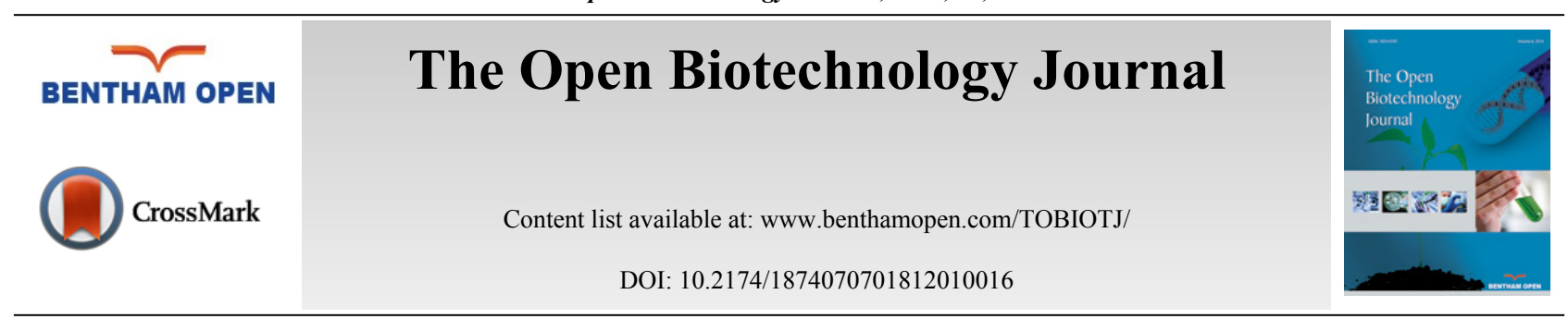

DOI: $10.2174 / 1874070701812010016$

\title{
Regulation of RNA Editing in Chloroplast
}

\author{
Wei Tang* \\ College of Horticulture and Gardening, Yangtze University, Jingzhou, Hubei 434025, China
}

Received: December 09, 2017

Revised: February 06, 2018

Accepted: February 08, 2018

\begin{abstract}
RNA editing is an important process involved in the modification of nucleotides in the transcripts of a large number of functional genes. RNA editing results in the restoration of conserved amino acid residues for protein function in plants. In this review, I only describe and discuss the identified RNA editing and the RNA editing associated regulation in chloroplast, including cytidine-to-uridine editing, adenosine-to-inosine editing, and regulation of RNA editing in model plants, crop plants, woody plants, and medical plants. Information described in this review could be valuable in future investigation of molecular mechanisms that determine the specificity of the RNA editing process.
\end{abstract}

Keywords: Adenosine-to-inosine editing, Chloroplasts, Cytidine-to-uridine editing, Pentatricopeptide repeat protein, RNA editing factor.

\section{INTRODUCTION}

Despite great progress has been made in technologies of analyzing RNA editing, critical editing factors remain to be identified in many of the plants [1]. RNA editing changes the sequence of transcripts of functional gens, the molecular mechanisms determining the specificity of the RNA editing process are not fully understood [2]. In plants, RNA editing factors and sequence-specific RNA maturation factors promote RNA editing and translatability [3]. Two types of RNA editing: cytidine-to-uridine editing in mRNAs and adenosine-to-inosine editing, have been identified in the model plant Arabidopsis thaliana [4]. RNA editing is a relatively rare molecular process that changes nucleotide sequences of a RNA molecule through the insertion, deletion, and base substitution of nucleotides within the edited RNA molecule under the function of RNA polymerase [5 - 8]. RNA editing has been reported in some tRNA, rRNA, and mRNA. However, RNA editing has also been discovered in non-coding RNA, such as microRNAs (miRNAs) molecules of eukaryotes. Micro RNAs are a large family of small RNAs with important functional roles in regulating gene expression in animals and plants [9 - 12]. In human and mouse, miRNA22 precursor molecules are subject to posttranscriptional modification by A-to-I RNA editing in vivo. A-to-I RNA editing of miRNA22 is predicted to have significant implications for the biogenesis and function of miRNA22. RNA editing of miRNA gene products could also take place in plants. In Arabidopsis, RNA editing in the precursor microRNAs of ath-miR854 family has a potential role in miRNA maturation [13 - 15].

Although RNA editing is an essential post-transcriptional modification in chloroplast gene expression, the factors mediating those processes are not fully identified in plants. It has been reported that the large Pentatricopeptide Repeat (PPR) protein family are required for RNA editing in chloroplasts [5].Chloroplasts contain their own genetic system. Most chloroplast genes are organized in clusters and are co-transcribed. Posttranscriptional RNA editing of chloroplast gene transcripts is an important step in the control of chloroplast gene expression and is required for gene function [6]. Considering RNA editing of nuclear gene transcripts has been widely reviewed, here I only review RNA editing and the RNA editing associated regulation in chloroplast (Table 1).

\footnotetext{
* Address correspondence to this author at the College of Horticulture and Gardening, Yangtze University, Jingzhou, Hubei 434025, China; Tel: +86-139-2107-4062; Fax: +86-716-806-6262; E-mail: wt10yu604@gmail.com
} 
Table 1. RNA Editing in chloroplasts of different plant species.

\begin{tabular}{|c|c|c|c|c|}
\hline Plant Species & Editing Type & Editing Factors & Edited Transcripts & Reference \\
\hline \multirow[t]{8}{*}{ Arabidopsis } & \begin{tabular}{|l|l} 
C-to-U & \\
\end{tabular} & CLB19 & rpoA and clpP & {$[2]$} \\
\hline & C-to-U & OTP80, OTP81, OTP85, and OTP86 & CRR22, CRR28, CLB19, OTP82, and OTP84, & {$[15]$} \\
\hline & A-to-I & tRNA adenosine deaminase arginine (TADA) & Chloroplast transcripts & {$[4]$} \\
\hline & C-to-U & CRR22 and CRR28 & Organelle transcripts & {$[16]$} \\
\hline & C-to-U & Quintuple editing factor 1 (QED1) and RARE1 & Chloroplast $\mathrm{C}$ targets & {$[23]$} \\
\hline & C-to-U & PDM1/SEL1 MORF9, MORF2, and MORF8 & Transcripts of trnK, ndhA, and accD-1 & {$[24]$} \\
\hline & C-to-U & $\begin{array}{l}\text { PDM2, organellar RNA editing factor } 2 \\
\text { (MORF2), and MORF9 }\end{array}$ & Chloroplast transcripts & {$[26]$} \\
\hline & C-to-U & $\begin{array}{l}\text { ORRM6, RIP1/MORF8, RIP2/MORF2, and } \\
\text { RIP9/MORF9 }\end{array}$ & psbF-C77 and accD-C794 & {$[25,26]$} \\
\hline \multirow[t]{5}{*}{ Tobacco } & C-to-U & Pentatricopeptide repeat protein & $\mathrm{rpoB}, \mathrm{psbL}$, and rps14 & {$[9,32]$} \\
\hline & C-to-U & Pentatricopeptide repeat protein & Plastid ATPase alpha-subunit transcript & {$[31]$} \\
\hline & C-to-U & Pentatricopeptide repeat protein & RNA-dependent RNA polymerase (RdRp) & {$[30]$} \\
\hline & C-to-U & Pentatricopeptide repeat protein & psaC and ndhD & {$[28,29]$} \\
\hline & C-to-U & Pentatricopeptide repeat protein & The asRNA and ndhB transcripts & {$[26,36]$} \\
\hline Poplar & C-to-U & Pentatricopeptide repeat protein & The asRNA_ndhB transcripts & {$[11]$} \\
\hline Spinach & C-to-U & Pentatricopeptide repeat protein & psbE, psbF, psbL and psbJ transcripts & {$[11]$} \\
\hline Pea & C-to-U & Pentatricopeptide repeat protein & Acetyl coA carboxylase gene transcripts & {$[10]$} \\
\hline \multirow[t]{2}{*}{ Rice } & C-to-U & CLB19, CRR4 and CRR21 & Transcripts of rpoA and clpP & {$[2]$} \\
\hline & C-to-U & CRR4, CRR21, and CLB19 & Acetyl coA carboxylase gene transcripts & {$[10,33]$} \\
\hline Potato & C-to-U & CLB19, RpoA, and ClpP1 & Transcript of accD & {$[33,34]$} \\
\hline Maize & C-to-U & Pentatricopeptide repeat protein & Transcript ofchloroplast ATP synthase gene & {$[5,34]$} \\
\hline Sweet potato & C-to-U & Pentatricopeptide repeat protein & Chloroplast functional-gene transcript & {$[34]$} \\
\hline Bell pepper & C-to-U & Pentatricopeptide repeat protein & Transcripts of the plastid psbL gene & {$[27,36]$} \\
\hline Mustard & C-to-U & Pentatricopeptide repeat protein & psaA transcripts & {$[37]$} \\
\hline Tomato & C-to-U & Pentatricopeptide repeat protein & accD transcripts & {$[38]$} \\
\hline Larix decidua & C-to-U & Pentatricopeptide repeat protein & Protochlorophyllide oxidoreductase & {$[40]$} \\
\hline Pinus sylvestris & C-to-U & Pentatricopeptide repeat protein & The chlB transcript & {$[39]$} \\
\hline Picea abies & C-to-U & Pentatricopeptide repeat protein & $\begin{array}{l}\text { Protochlorophyllide oxidoreductase gene } \\
\text { transcripts }\end{array}$ & {$[40]$} \\
\hline Dianthus superbus & C-to-U & Pentatricopeptide repeat protein & Transcripts of ribosomal protein subunit S19 & {$[44]$} \\
\hline $\begin{array}{l}\text { Chlamydomonas } \\
\text { reinhardtii }\end{array}$ & C-to-U & OPR, MCG1, and MBI1 & petG transcript, PsbI transcripts & {$[45]$} \\
\hline Rhazya stricta & C-to-U & $\begin{array}{l}\text { Tetrapyrroles and pentatricopeptide repeat } \\
\text { proteins }\end{array}$ & $\begin{array}{l}\text { Dnaj6, UDP-glucosyl transferase 85a2 gene } \\
\text { transcripts }\end{array}$ & {$[46]$} \\
\hline
\end{tabular}

\section{RNA EDITING IN CHLOROPLAST}

\subsection{C-to-U Editing}

RNA editing is an important process of gene regulation through nucleotide modification at post-transcriptional level. Many transcripts expressed in chloroplasts are modified by C-to-U RNA editing. C-to-U editing is the major type of RNA editing discovered in the chloroplasts of plants including Arabidopsis, tobacco, maize, rice, and potato. In maize chloroplasts, 27 C-to-U RNA editing sites have been identified to affect the expression of at least 15 different genes in 10 different maize tissues including chloroplasts, etioplasts, and amyloplasts. The role of editing in chloroplasts is to correct detrimental mutations rather than to produce protein diversity [7]. Although C-to-U editing modifies the chloroplast genes in maize, the mechanism for recognition of the targets of editing is not fully understood [8]. It has been reported that several nuclear-encoded proteins containing pentatricopeptide repeat motifs have been essential for chloroplast RNA editing through analysis of mutants affected in chloroplast biogenesis in Arabidopsis and rice. Many PPR proteins include a C-terminal DYW deaminase domain and the zinc binding motifs [9]. For example, the Arabidopsis nuclear-encoded gene RARE1 is required for editing of the chloroplast accD transcript [10]. The DYW deaminase domains of PPR proteins are involved in editing their cognate editing sites in chloroplasts [9]. C-to-U editing modification does not change the genome DNA sequence. RNA editing changes specific nucleotide sequences within a RNA molecule. RNA editing has generated by RNA polymerase [7, 8, 10]. RNA editing in mRNAs effectively alters 
the amino acid sequence of the encoded protein so that it differs from that predicted by the genomic DNA sequence. However, RNA editing including C-to-U editing modification does not change the genome DNA sequence. The events of RNA editing include the insertion, deletion, and base substitution of nucleotides within the edited RNA molecule. Therefore, RNA editing can serves as a safeguard because it can reverse harmful genomic mutations in corresponding RNA transcripts. Genomic studies in humans have uncovered a large number of RNA editing sites. Because cells employ RNA editing mechanisms to correct mistakes made during DNA replication, RNA editing can mean correcting detrimental mutations.

Computational prediction of RNA secondary structures in chloroplasts of Arabidopsis, Nicotiana tabacum, and poplar identified a long antisense RNA that is typical for the plastid-encoded RNA polymerase gene ndhB. The asRNA_ndhB transcripts accumulate in young leaves and are subject to C-to-U RNA editing [11]. In some cases, C-toU RNA editing enzymes may participate in editing different sites in the chloroplast [9]. In the chloroplast DNA of moth orchid (P. aphrodite subsp. Formosana), 137 edits including $126 \mathrm{C}$-to-U and $11 \mathrm{U}$-to-C conversions were identified. 110 and 106 edits were present in leaf and floral tissues, respectively. RNA editing occurred in non-protein-coding transcripts such as tRNA, introns and some regulatory regions [12].

\subsection{A-to-I Editing}

Although cytidine-to-uridine editing has been reported to be the majority of RNA editing in the chloroplasts of plants, another type of RNA editing: adenosine-to-inosine editing in a plastid genome-encoded tRNA has been characterized in the chloroplasts of the model plant Arabidopsis thaliana. A-to-I editing was identified in the anticodon of the plastid tRNA-Arg(ACG). AtTadA gene expression is involved in A-to-I editing in the chloroplast [4].

\section{RNA EDITING IN MODEL PLANTS}

\subsection{Arabidopsis}

A large number of nucleus-encoded factors discovered via biochemical approaches in Arabidopsis may regulate plastid gene expression through RNA editing. The nucleus-encoded factors may modulate chloroplast gene expression in response to environmental cues via the assembly of the protein products into the photosynthetic apparatus [3]. The discovery of genes responsible for the specific RNA editing in the chloroplast via genetic approach is important in Arabidopsis [1]. The pentatricopeptide repeat proteins CLB19, CRR4, and CRR21 are required for editing of chloroplast transcripts rpoA and clpP that are important in chloroplast development and early seedling development [2]. Using the transgenomic suppression of point mutations, additional nuclear-encoded components have been found in chloroplast, indicating that several chloroplast-specific mechanisms evolved in the regulatory functions of the complexity of chloroplast gene expression in Arabidopsis [13]. Biochemical and mutant complementation studies showed that the Arabidopsis thaliana tRNA adenosine deaminase arginine (TADA) was required for deamination of chloroplast (cp)-tRNAArg(ACG) to cp-tRNAArg (ICG) and disruption of TADA reduced yields of chloroplast-encoded proteins and impaired photosynthetic function [14].

Analysis of mutants in chloroplast biogenesis resulted in the identification of several Pentatricopeptide Repeat (PPR) proteins that are essential for the editing of RNA transcripts in Arabidopsis thaliana. The editing factors OTP80, OTP81, OTP82, OTP84, OTP85, and OTP86 participate in more than 17 editing events and are involved in the editing of multiple sites CRR22, CRR28, CLB19, OTP82, and OTP84 in Arabidopsis chloroplasts [15]. A-to-I editing in the anticodon of the plastid tRNA-Arg(ACG) reduced chloroplast translational efficiency [4]. Different DYW family members have different functions in RNA editing. The DYW proteins CHLORORESPIRATORY REDUCTION22 and CRR28 are necessary for editing of multiple plastid transcripts [16]. Chloroplast ribonucleoproteins (cpRNPs) are involved in chloroplast RNA processing in chloroplasts and the cpRNP family member CP31A exhibits highly specific defects in chloroplast RNA metabolism, suggesting that these chloroplast proteins are functional equivalents of nucleocytosolic hnRNPs [17]. The AtECB2 gene codes a pentatricopeptide repeat protein with a C-terminal DYW domain that is required for chloroplast transcript accD RNA editing and early chloroplast biogenesis in Arabidopsis thaliana [18].

RNA editing sites found in chloroplasts of Arabidopsis could be involved in RNA maturation [11]. The editing of accD and ndhF transcripts is partially affected by the vanilla cream1 (vac1) albino mutant, suggesting that the VAC1 protein may be involved in plastid-to-nucleus retrograde signaling in addition to its role in chloroplast RNA editing and gene expression [19]. Functional significance of these processing events is an active area of current research [20]. SEL1 
is a pentatricopeptide repeat gene that is involved in the regulation of plastid gene expression required for normal chloroplast development [21]. SVR7 gene that encodes a PPR-SMR protein in Arabidopsis thaliana is involved in translational activation of chloroplast ATP synthase in eudicotyledonous and monocotyledonous plants [5]. Many PPR proteins with a C-terminal DYW deaminase domain and zinc binding motifs are required in C-to-U editing. For example, OTP84 is required for editing three chloroplast sites in Arabidopsis thaliana [9].

CLB19 is essential for the editing and functionality of the subunit A of plastid-encoded RNA polymerase (RpoA) and the catalytic subunit of the Clp protease (ClpP1) [22]. The chloroplast PPR-DYW editing factor, Quintuple Editing factor 1 (QED1), was shown to affect five different plastid editing sites and is required for editing efficiency [23]. PDM1 interacts directly with MORF9, MORF2, and MORF8 to edit the transcripts of trnK and ndhA, as well as accD-1, suggesting that PDM1 is an important protein for post-transcriptional regulation in chloroplast [24]. PDM2 that encodes a pentatricopeptide repeat protein that interacts with Multiple Organellar RNA editing Factor 2 (MORF2) and MORF9 to affect plastid RNA editing efficiency [25]. ORRM6 (ORGANELLE RNA RECOGNITION MOTIF PROTEIN6) interacts with RIP1/MORF8, RIP2/MORF2, and RIP9/MORF9 to regulate RNA editing of psbF-C77 and accD-C794 in Arabidopsis [26].

Mutation stress, DNA duplication, ABA stress, abiotic stresses such as salt, drought, and low temperature stress can induce Pentatricopeptide Repeat (PPR) proteins [1, 2, 25]. Horizontal gene transfer has played a major role in the sporadic phylogenetic distribution of different PPR subclasses in both eukaryotes and prokaryotes. Pentatricopeptide repeat (PPR) proteins are a large family of RNA-binding proteins that regulate gene expression in organelles and the nucleus $[1,25]$. PPRs have functions in RNA processing, splicing, editing, stability and translation. PPR proteins are classified into different subclasses based on their domain architecture and their function. Investigation into the structure and function of PPR-TGM proteins presents a novel opportunity for the exploitation of PPR proteins as drug targets to prevent disease $[2,25]$. Functional and evolutionary studies provide insights into the different PPR subfamilies and their phylogenetic distributions will facilitate the understanding of the eukaryotic PPR protein families identified to date. However, mitochondrial and chloroplast Pentatricopeptide Repeat (PPR) proteins have been reported to regulate plant response to abiotic stresses $[1,2,25]$.

\subsection{Tobacco}

RNA editing alters the transcript levels of tobacco chloroplast genes. Sequence analysis revealed $13 \mathrm{C}$-to-U editing sites in transcripts of 11 tobacco chloroplast genes. For example, the genes psbE, psbF, psbL and psbJ, encode two RNA editing sites. RNA editing process is differentially down-regulated in leucoplasts and proplastids and may function as a regulatory device in plastid gene expression [27]. In tobacco chloroplasts, RNA factors and RNA-binding proteins are involved in RNA editing and the regulation of gene expression in chloroplasts [6]. Transcripts of two tobacco chloroplast genes, the photosystem I component (psaC) and the NADH dehydrogenase subunit (ndhD), are modified through C-to-U editing [28]. Editing in tobacco rpoA mRNA restores the conserved leucine residue which is known to be important for transcriptional activation, suggesting that editing may be involved in the regulation of chloroplast-encoded RNA polymerase activity [29]. In the ndhD transcript of tobacco, editing of an ACG codon to a standard AUG initiator codon is a prerequisite for translation. The presence of an RNA-dependent RNA polymerase $(\mathrm{RdRp})$ activity may have general implications in plastid gene expression [30].

The expression of tobacco chloroplast genes is modified by C-to-U RNA editing. It is not known whether complementary RNA is involved in chloroplast editing site recognition. Expressing RNA antisense to the sequences -20 to +6 surrounding the RpoB-2 $\mathrm{C}$ target of editing had shown that transcripts carrying sequences -31 to +60 surrounding the RpoB-2 sites were edited in chloroplast transgenic plants [8]. A defect in RNA editing of a tobacco-specific editing site in the plastid ATPase alpha-subunit transcript results in the albino phenotype of Nicotiana tabacum, suggesting that differences in RNA editing patterns contribute to the pigment deficiencies [31]. In tobacco chloroplast transcripts $34 \mathrm{nt}$ are efficiently edited to U. Transgene transcripts carrying either the wild-type $-31 /+22$ or $-31 /+60$ sequence near NTrpoB C473, an editing site within tobacco rpoB transcripts, were highly edited in vivo and the transcripts of rpoB, psbL and rps14 are critical for efficient NTrpoB C473 editing [32]. Two nucleotide positions in the asRNA_ndhB transcripts that predominantly accumulate in young leaves are subject to C-to-U RNA editing and the accumulation of asRNA_ndhB and RNA editing appeared weak in a temperature shift experiment, suggesting that long asRNAs could be involved in RNA stability [11]. 


\section{RNA EDITING IN CROP PLANTS}

\subsection{Rice}

RNA editing changes the sequence of many transcripts in rice. The rice CLB19, which encodes a pentatricopeptide repeat protein, edits two chloroplast transcripts, rpoA and clpP [2]. Biochemical studies showed that the C-terminal domain is sufficient for tRNA deamination in plants [14]. Proteins containing pentatricopeptide repeat motifs have been identified to be trans-factors essential for chloroplast RNA editing in rice. Rice contains over 400 PPR genes including 80 C-terminal DYW domains. The rice editing factors CRR4, CRR21, and CLB19 are required for editing of the chloroplast accD transcript [10]. Gene expression in nongreen plastids is largely uncharacterized. Transcripts of photosynthesis-related genes showed a greater reduction in nongreen plastids compared with leaves. The transcripts of the fatty acid biosynthesis gene accD, displayed relatively high ribosome association [33]. Results of site-directed mutagenesis of the rpoA target demonstrated that the E domain of CLB19 interacts with the RNA-interacting protein MORF2/RIP2 for the editing and functionality of the subunit A of plastid-encoded RNA polymerase (RpoA) [22].

\subsection{Maize}

The expression of the maize plastid genome is dependent on many nucleus-encoded factors. The maize nucleusencoded factors function in plastid gene expression and RNA editing [3]. In maize plastids, transcripts are modified at $27 \mathrm{C}$-to-U RNA editing sites and expression of 15 different genes is affected. The editing efficiency of different sites postulates individual trans-acting factors specific to each editing site [7]. RNA editing is an essential posttranscriptional step in chloroplast gene expression. The pentatricopeptide repeat proteins are required for chloroplast RNA editing. For example, maize ATP4 gene functions in maize chloroplast RNA editing [5]. The organization and structure of the chloroplast genome of maize were compared with other species and some gene gain-and-loss events were identified. In addition, the RNA editing events and differential expressions of the chloroplast functional-genes were detected [34].

\subsection{Bell Pepper}

The nucleotide sequence of the plastid psbL gene from bell pepper is an example of RNA editing. Sequencing of the psbL cDNA revealed that ACG codon is post-transcriptionally edited into an AUG initiation codon in leaves, indicating that the RNA editing machinery exists in bell pepper chloroplasts [35]. RNA editing in chloroplasts proceeds by the conversion of individual cytidine residues to uridine. The discovery of RNA editing in chloroplasts has provided researchers with a wealth of molecular puzzles and with extraordinarily high precision [36].

\subsection{Mustard}

The mustard chloroplast ycf3 gene was investigated for RNA editing. The ycf3 gene revealed two class-II introns that were removed, but no RNA editing seemed to be involved. Whereas transcripts of ycf3 and psaA initiated from PEP promoters, as well as transcripts of photosystem core-protein genes were edited [37].

\subsection{Tomato}

Transcriptomics and translatomics analysis of the tomato (Solanum lycopersicum) plastid genome demonstrated that RNA editing exists in plastid genes in fruits and leaves. Sequencing analysis during chromoplast development demonstrated that transcripts of the plastid-encoded gene, accD, which is involved in fatty acid biosynthesis, has the developmental patterns of RNA editing through specific developmental changes in RNA editing efficiency [38].

\section{RNA EDITING IN WOODY PLANTS}

\subsection{Pinus sylvestris}

RNA editing was less investigated in gymnosperms than in angiosperms. In pine species, RNA editing was first identified in the chlB transcript of Pinus sylvestris. ChlB is a chloroplast gene required for light-independent chlorophyll synthesis. Two C-to-U editing sites have been identified in the transcripts of ChIB. The editing of a CCG codon leads to an amino-acid substitution from proline to leucine and the editing of a CGG codon results in an arginine to tryptophan substitution [39]. 


\subsection{Larix decidua}

Light-independent Chlorophyll (Chl) biosynthesis is important for the photosynthetic pigment-protein complexes. Expression of $\mathrm{CHl}$ genes is regulated by RNA editing. In Larix decidua Mill seedlings, C-to-U RNA editing exists in the transcripts of the $\mathrm{Chl}$ biosynthesis genes chlL, chlN and chlB that encode subunits of the light-independent protochlorophyllide oxidoreductase. The accumulation of the ChlB subunit was developmentally regulated and the efficiency of chlB RNA-editing was reduced in the mature dark-grown larch seedlings. RNA editing was also identified in seedlings of Picea abies (L.) Karst [40]. Expression of plastid genes showed organelle-specific elaborations on a prokaryotic scaffold [41]. The nucleus provides essential factors that are involved in many processes inside the chloroplast including RNA editing that regulates the expression and assembly of the photosynthetic thylakoid membrane complexes [42]. Expression of most plastid genes involves specific post-transcriptional processing events, such as RNA editing. Expression of the psbB gene cluster and RNA editing factors stabilize many of the processed RNA transcripts in the chloroplast [43].

\section{RNA EDITING IN MEDICAL PLANTS}

\subsection{Dianthus superbus}

RNA editing has been identified in a few of medical plants. In Dianthus superbus var. longicalycinus, the ribosomal protein subunit S19 (rps19), the translation initiation factor IF-1 (infA), and ribosomal protein subunit L23 (rpl23) genes were contributed to the molecular biology and genetic engineering of RNA editing [44].

\subsection{Chlamydomonas reinhardtii}

RNA editing of transcripts of chloroplast genes has been identified in Chlamydomonas reinhardtii. MCG1 and MBI1, which code for subunit of photosystem II, are editing related factors that are essential for functional cytochrome b6f dimer and are involved in translation of plastid mRNAs [45].

\subsection{Rhazya stricta}

A large number of transcripts including Pentatricopeptide Repeat (PPR) protein genes have been identified in Rhazya stricta through RNA-Seq analysis. Chaperone protein Dnaj6 and protein transparent testa 12 are related to abiotic responses and participate Reactive Oxygen Species (ROS) production. They are targeted editing genes of the PPR gene family that were independent of the salt stress due to their RNA editing patterns were unchanged [46].

\section{CONCLUSION}

We have overviewed the identified RNA editing and the RNA editing associated regulation in chloroplast, including cytidine-to-uridine editing, adenosine-to-inosine editing, and regulation of RNA editing in model plants, crop plants, woody plants, and medical plants. RNA editing is conducted by an editing complex called editosome that includes the deaminase [29, 32, 36]. The mechanism of the editosome includes an endonucleolytic cut at the mismatch point between the guide RNA and the unedited transcript. After the endonucleolytic cut, a terminal U-transferase adds U from UTP at the 3' end of the mRNA and a U-specific exoribonuclease removes the unpaired base, followed by an RNA ligase to rejoin the ends of the edited mRNA transcript. The RNA editing complex can use only a single guide RNA at a time $[29,36]$. Therefore, more than one guide RNA and editosome complex will be needed when a RNA transcript requires extensive editing. The RNA editing process has been observed in some tRNA, rRNA, mRNA, or miRNA molecules of eukaryotes [32, 36].

RNA editing occurs in the cell nucleus and cytosol, as well as within mitochondria and plastids. Editing of mRNA or other kinds of RNA is made to individual nucleotides by changing the base-pairing potential through the process of translation that involves base pairing between mRNA and tRNAs [29, 32, 36]. Editing of the mRNA occurs at the 3' end of the mRNA and may change the amino acid of the protein to increase the diversity of protein products that can be synthesized from the genome [29, 32]. Cells may also use different mechanisms that insert and delete nucleotides from RNAs in some eukaryotes. An example is in the mitochondria of trypanosomes [29, 36]. Uracil residues are added, or deleted from the mitochondrial mRNAs at many sites through the interaction of a large number of small guide RNAs. The best-characterized example of C-to-U RNA editing is the editing of the mRNA of apolipoprotein B that generates a stop codon and leads to down-regulation of protein expression [29, 32, 36].

RNA editing occurs during the process of transcription and the process of mRNA splicing in the nucleus, 
chloroplasts, or mitochondria [32, 36]. The process of RNA editing comes in a myriad of different biological and evolutional natures across the phylogenetic spectrum. RNA editing is a biochemical process that cells can make discrete changes to specific nucleotide sequences within a RNA molecule [29, 36]. RNA editing makes distinct changes to RNA after its synthesis and before the translation of the mRNA into a protein. In addition to specific editing of individual nucleotides in the nucleus, mitochondria, and plastids, RNA editing is also a dynamic mechanism that generates molecular and functional diversity $[29,32,36]$. Knowledge described in this review could be valuable in determining the specificity of the RNA editing process.

\section{CONSENT FOR PUBLICATION}

Not applicable.

\section{CONFLICT OF INTEREST}

The authors declare no conflict of interest, financial or otherwise.

\section{ACKNOWLEDGEMENTS}

The authors are grateful to Wells Thompson for critical reading of the manuscript. This research was supported the National Natural Science Foundation of China (31270740).

\section{REFERENCES}

[1] Kotera E, Tasaka M, Shikanai T. A pentatricopeptide repeat protein is essential for RNA editing in chloroplasts. Nature 2005; 433(7023): 326-30.

[http://dx.doi.org/10.1038/nature03229] [PMID: 15662426]

[2] Chateigner-Boutin AL, Ramos-Vega M, Guevara-García A, et al. CLB19, a pentatricopeptide repeat protein required for editing of rpoA and clpP chloroplast transcripts. Plant J 2008; 56(4): 590-602. [http://dx.doi.org/10.1111/j.1365-313X.2008.03634.x] [PMID: 18657233]

[3] Barkan A, Goldschmidt-Clermont M. Participation of nuclear genes in chloroplast gene expression. Biochimie 2000; 82(6-7): 559-72. [http://dx.doi.org/10.1016/S0300-9084(00)00602-7] [PMID: 10946107]

[4] Karcher D, Bock R. Identification of the chloroplast adenosine-to-inosine tRNA editing enzyme. RNA 2009; $15(7)$ : $1251-7$. [http://dx.doi.org/10.1261/rna.1600609] [PMID: 19460869]

[5] Zoschke R, Qu Y, Zubo YO, Börner T, Schmitz-Linneweber C. Mutation of the pentatricopeptide repeat-SMR protein SVR7 impairs accumulation and translation of chloroplast ATP synthase subunits in Arabidopsis thaliana. J Plant Res 2013; 126(3): 403-14. [http://dx.doi.org/10.1007/s10265-012-0527-1] [PMID: 23076438]

[6] Sugita M, Sugiura M. Regulation of gene expression in chloroplasts of higher plants. Plant Mol Biol 1996; 32 (1-2): 315-26. [http://dx.doi.org/10.1007/BF00039388] [PMID: 8980485]

[7] Peeters NM, Hanson MR. Transcript abundance supercedes editing efficiency as a factor in developmental variation of chloroplast gene expression. RNA 2002; 8(4): 497-511

[http://dx.doi.org/10.1017/S1355838202029424] [PMID: 11991643]

[8] Hegeman CE, Halter CP, Owens TG, Hanson MR. Expression of complementary RNA from chloroplast transgenes affects editing efficiency of transgene and endogenous chloroplast transcripts. Nucleic Acids Res 2005; 33(5): 1454-64. [http://dx.doi.org/10.1093/nar/gki286] [PMID: 15755747]

[9] Hayes ML, Dang KN, Diaz MF, Mulligan RM. A conserved glutamate residue in the C-terminal deaminase domain of pentatricopeptide repeat proteins is required for RNA editing activity. J Biol Chem 2015; 290(16): 10136-42. [http://dx.doi.org/10.1074/jbc.M114.631630] [PMID: 25739442]

[10] Robbins JC, Heller WP, Hanson MR. A comparative genomics approach identifies a PPR-DYW protein that is essential for C-to-U editing of the Arabidopsis chloroplast accD transcript. RNA 2009; 15(6): 1142-53. [http://dx.doi.org/10.1261/rna.1533909] [PMID: 19395655]

[11] Georg J, Honsel A, Voss B, Rennenberg H, Hess WR. A long antisense RNA in plant chloroplasts. New Phytol 2010; 186(3): 615-22. [http://dx.doi.org/10.1111/j.1469-8137.2010.03203.x] [PMID: 20202127]

[12] Chen TC, Liu YC, Wang X, Wu CH, Huang CH, Chang CC. Whole plastid transcriptomes reveal abundant RNA editing sites and differential editing status in Phalaenopsis aphrodite subsp. formosana. Bot Stud (Taipei, Taiwan) 2017; 58(1): 38 [http://dx.doi.org/10.1186/s40529-017-0193-7] [PMID: 28916985]

[13] Maier UG, Bozarth A, Funk HT, et al. Complex chloroplast RNA metabolism: just debugging the genetic programme? BMC Biol 2008 ; 6: 36. [http://dx.doi.org/10.1186/1741-7007-6-36] [PMID: 18755031]

[14] Delannoy E, Le Ret M, Faivre-Nitschke E, et al. Arabidopsis tRNA adenosine deaminase arginine edits the wobble nucleotide of chloroplast tRNAArg(ACG) and is essential for efficient chloroplast translation. Plant Cell 2009; 21(7): 2058-71. 
[http://dx.doi.org/10.1105/tpc.109.066654] [PMID: 19602623]

[15] Hammani K, Okuda K, Tanz SK, Chateigner-Boutin AL, Shikanai T, Small I. A study of new Arabidopsis chloroplast RNA editing mutants reveals general features of editing factors and their target sites. Plant Cell 2009; 21(11): 3686-99. [http://dx.doi.org/10.1105/tpc.109.071472] [PMID: 19934379]

[16] Okuda K, Chateigner-Boutin AL, Nakamura T, et al. Pentatricopeptide repeat proteins with the DYW motif have distinct molecular functions in RNA editing and RNA cleavage in Arabidopsis chloroplasts. Plant Cell 2009; 21(1): 146-56. [http://dx.doi.org/10.1105/tpc.108.064667] [PMID: 19182104]

[17] Tillich M, Hardel SL, Kupsch C, et al. Chloroplast ribonucleoprotein CP31A is required for editing and stability of specific chloroplast mRNAs. Proc Natl Acad Sci USA 2009; 106(14): 6002-7. [http://dx.doi.org/10.1073/pnas.0808529106] [PMID: 19297624]

[18] Yu QB, Jiang Y, Chong K, Yang ZN. AtECB2, a pentatricopeptide repeat protein, is required for chloroplast transcript accD RNA editing and early chloroplast biogenesis in Arabidopsis thaliana. Plant J 2009; 59(6): 1011-23. [http://dx.doi.org/10.1111/j.1365-313X.2009.03930.x] [PMID: 19500301]

[19] Tseng CC, Sung TY, Li YC, Hsu SJ, Lin CL, Hsieh MH. Editing of accD and ndhF chloroplast transcripts is partially affected in the Arabidopsis vanilla cream1 mutant. Plant Mol Biol 2010; 73(3): 309-23. [http://dx.doi.org/10.1007/s11103-010-9616-5] [PMID: 20143129]

[20] Barkan A. Studying the structure and processing of chloroplast transcripts. Methods Mol Biol 2011; 774: 183-97. [http://dx.doi.org/10.1007/978-1-61779-234-2_12] [PMID: 21822840]

[21] Pyo YJ, Kwon KC, Kim A, Cho MH. Seedling Lethal1, a pentatricopeptide repeat protein lacking an E/E+ or DYW domain in Arabidopsis, is involved in plastid gene expression and early chloroplast development. Plant Physiol 2013; 163(4): 1844-58. [http://dx.doi.org/10.1104/pp.113.227199] [PMID: 24144791]

[22] Ramos-Vega M, Guevara-García A, Llamas E, et al. Functional analysis of the Arabidopsis thaliana CHLOROPLAST BIOGENESIS 19 pentatricopeptide repeat editing protein. New Phytol 2015; 208(2): 430-41. [http://dx.doi.org/10.1111/nph.13468] [PMID: 25980341]

[23] Wagoner JA, Sun T, Lin L, Hanson MR. Cytidine deaminase motifs within the DYW domain of two pentatricopeptide repeat-containing proteins are required for site-specific chloroplast RNA editing. J Biol Chem 2015; 290(5): 2957-68. [http://dx.doi.org/10.1074/jbc.M114.622084] [PMID: 25512379]

[24] Zhang HD, Cui YL, Huang C, et al. PPR protein PDM1/SEL1 is involved in RNA editing and splicing of plastid genes in Arabidopsis thaliana. Photosynth Res 2015; 126(2-3): 311-21. [http://dx.doi.org/10.1007/s11120-015-0171-4] [PMID: 26123918]

[25] Du L, Zhang J, Qu S, et al. The pentratricopeptide repeat protein pigment-defective mutant2 is involved in the regulation of chloroplast development and chloroplast gene expression in arabidopsisplant cell Physio 2017; 58(2-3): 747-59. [http://dx.doi.org/10.1093/pcp/pcx004]

[26] Hackett JB, Shi X, Kobylarz AT, et al. An organelle RNA recognition motif protein Is required for photosystem II subunit psbF transcript editing. Plant Physiol 2017; 173(4): 2278-93. [PMID: 28213559]

[27] Bock R, Hagemann R, Kössel H, Kudla J. Tissue- and stage-specific modulation of RNA editing of the psbF and psbL transcript from spinach plastids--a new regulatory mechanism? Mol Gen Genet 1993; 240(2): 238-44. [http://dx.doi.org/10.1007/BF00277062] [PMID: 8355656]

[28] Hirose T, Sugiura M. Both RNA editing and RNA cleavage are required for translation of tobacco chloroplast ndhD mRNA: a possible regulatory mechanism for the expression of a chloroplast operon consisting of functionally unrelated genes. EMBO J 1997; $16(22)$ : $6804-11$. [http://dx.doi.org/10.1093/emboj/16.22.6804] [PMID: 9362494]

[29] Hirose T, Kusumegi T, Tsudzuki T, Sugiura M. RNA editing sites in tobacco chloroplast transcripts: editing as a possible regulator of chloroplast RNA polymerase activity. Mol Gen Genet 1999; 262(3): 462-7. [http://dx.doi.org/10.1007/s004380051106] [PMID: 10589833]

[30] Zandueta-Criado A, Bock R. Surprising features of plastid ndhD transcripts: addition of non-encoded nucleotides and polysome association of mRNAs with an unedited start codon. Nucleic Acids Res 2004; 32(2): 542-50. [http://dx.doi.org/10.1093/nar/gkh217] [PMID: 14744979]

[31] Schmitz-Linneweber C, Kushnir S, Babiychuk E, Poltnigg P, Herrmann RG, Maier RM. Pigment deficiency in nightshade/tobacco cybrids is caused by the failure to edit the plastid ATPase alpha-subunit mRNA. Plant Cell 2005; 17(6): 1815-28. [http://dx.doi.org/10.1105/tpc.105.032474] [PMID: 15894714]

[32] Hayes ML, Reed ML, Hegeman CE, Hanson MR. Sequence elements critical for efficient RNA editing of a tobacco chloroplast transcript in vivo and in vitro. Nucleic Acids Res 2006; 34(13): 3742-54. [http://dx.doi.org/10.1093/nar/gk1490] [PMID: 16893957]

[33] Valkov VT, Scotti N, Kahlau S, et al. Genome-wide analysis of plastid gene expression in potato leaf chloroplasts and tuber amyloplasts: transcriptional and posttranscriptional control. Plant Physiol 2009; 150(4): 2030-44. [http://dx.doi.org/10.1104/pp.109.140483] [PMID: 19493969] 
[34] Yan L, Lai X, Li X, Wei C, Tan X, Zhang Y. Analyses of the complete genome and gene expression of chloroplast of sweet potato [Ipomoea batata]. PLoS One 2015; 10(4): e0124083. [Ipomoea batata]. [http://dx.doi.org/10.1371/journal.pone.0124083] [PMID: 25874767]

[35] Kuntz M, Camara B, Weil JH, Schantz R. The psbL gene from bell pepper (Capsicum annuum): plastid RNA editing also occurs in nonphotosynthetic chromoplasts. Plant Mol Biol 1992; 20(6): 1185-8. [http://dx.doi.org/10.1007/BF00028906] [PMID: 1463853]

[36] Bock R. Sense from nonsense: how the genetic information of chloroplasts is altered by RNA editing. Biochimie 2000; 82(6-7): 549-57. [http://dx.doi.org/10.1016/S0300-9084(00)00610-6] [PMID: 10946106]

[37] Summer H, Pfannschmidt T, Link G. Transcripts and sequence elements suggest differential promoter usage within the ycf3-psaAB gene cluster on mustard (Sinapis alba L.) chloroplast DNA. Curr Genet 2000; 37(1): 45-52. [http://dx.doi.org/10.1007/s002940050007] [PMID: 10672444]

[38] Kahlau S, Bock R. Plastid transcriptomics and translatomics of tomato fruit development and chloroplast-to-chromoplast differentiation: chromoplast gene expression largely serves the production of a single protein. Plant Cell 2008; 20(4): 856-74. [http://dx.doi.org/10.1105/tpc.107.055202] [PMID: 18441214]

[39] Karpinska B, Karpinski S, Hällgren J. The chlB gene encoding a subunit of light-independent protochlorophyllide reductase is edited in chloroplasts of conifers. Curr Genet 1997; 31(4): 343-7. [http://dx.doi.org/10.1007/s002940050214] [PMID: 9108142]

[40] Demko V, Pavlovic A, Valková D, Slováková L, Grimm B, Hudák J. A novel insight into the regulation of light-independent chlorophyll biosynthesis in Larix decidua and Picea abies seedlings. Planta 2009; 230(1): 165-76. [http://dx.doi.org/10.1007/s00425-009-0933-3] [PMID: 19404675]

[41] Barkan A. Expression of plastid genes: organelle-specific elaborations on a prokaryotic scaffold. Plant Physiol 2011; 155(4): 1520-32. [http://dx.doi.org/10.1104/pp.110.171231] [PMID: 21346173]

[42] Lyska D, Meierhoff K, Westhoff P. How to build functional thylakoid membranes: from plastid transcription to protein complex assembly. Planta 2013; 237(2): 413-28.

[http://dx.doi.org/10.1007/s00425-012-1752-5] [PMID: 22976450]

[43] Stoppel R, Meurer J. Complex RNA metabolism in the chloroplast: an update on the psbB operon. Planta 2013; 237(2): 441-9. [http://dx.doi.org/10.1007/s00425-012-1782-z] [PMID: 23065055]

[44] Raman G, Park S. Analysis of the complete chloroplast genome of a medicinal plant, Dianthus superbus var. longicalyncinus, from a comparative genomics perspective. PLoS One 2015; 10(10): e0141329. [http://dx.doi.org/10.1371/journal.pone.0141329] [PMID: 26513163]

[45] Wang F, Johnson X, Cavaiuolo M, Bohne AV, Nickelsen J, Vallon O. Two Chlamydomonas OPR proteins stabilize chloroplast mRNAs encoding small subunits of photosystem II and cytochrome b6 f. Plant J 2015; 82(5): 861-73. [http://dx.doi.org/10.1111/tpj.12858] [PMID: 25898982]

[46] Hajrah NH, Obaid AY, Atef A, et al. Transcriptomic analysis of salt stress responsive genes in Rhazya stricta. PLoS One 2017; 12(5): e0177589.

[http://dx.doi.org/10.1371/journal.pone.0177589] [PMID: 28520766]

\section{(C) 2018 Wei Tang.}

This is an open access article distributed under the terms of the Creative Commons Attribution 4.0 International Public License (CC-BY 4.0), a copy of which is available at: (https://creativecommons.org/licenses/by/4.0/legalcode). This license permits unrestricted use, distribution, and reproduction in any medium, provided the original author and source are credited. 